\title{
Effects of $A G X T 2$ variants on blood pressure and blood sugar among 750 older Japanese subjects recruited by the complete enumeration survey method
}

Yuta Yoshino', Hiroshi Kumon', Takaaki Mori ${ }^{1}$, Taku Yoshida², Ayumi Tachibana', Hideaki Shimizu', Jun-ichi $\lg ^{1 *}$ and Shu-ichi Ueno ${ }^{1}$

\begin{abstract}
Background: Alanine:glyoxylate aminotransferase 2 (AGXT2; EC 2.6.1.44) is the only enzyme that degrades the R-form of 3-aminoisobutyrate, an intermediate metabolite of thymine. AGXT2, as well as diaminoarginine dimethylaminohydrolase 1 (DDAH1; EC 3.5.3.18), works as an enzyme that degrades asymmetric dimethylarginine (ADMA), which competitively inhibits the nitric oxide synthase family. Thus, these two enzyme activities may change vascular vulnerability for a lifetime via the nitric oxide (NO) system. We investigated the association between vascular conditions and diseases such as hypertension and diabetes mellitus and polymorphisms of these two genes in 750 older Japanese subjects (mean age \pm standard deviation, $77.0 \pm 7.6$ years) recruited using the complete enumeration survey method in the Nakayama study. Demographic and biochemical data, such as blood pressure (BP) and casual blood sugar (CBS), were obtained. Four functional single nucleotide polymorphisms (SNPs; rs37370, rs37369, rs180749, and rs16899974) of AGXT2 and one functional insertion/ deletion polymorphism in the promotor region with four SNPs (rs307894, rs669173, rs997251, and rs13373844) of DDAH1 were investigated. Plasma ADMA was also analyzed in 163 subjects.
\end{abstract}

Results: The results of multiple regression analysis showed that a loss of the functional haplotype of AGXT2, CAAA, was significantly positively correlated with BP (systolic BP, $p=0.034$; diastolic BP, $p=0.025$ ) and CBS $(p=0.021$ ). No correlation was observed between DDAH1 and either BP or CBS. ADMA concentrations were significantly elevated in subjects with two CAAA haplotypes compared with subjects without the CAAA haplotype $(p=0.033)$.

Conclusions: Missense variants of AGXT2, but not DDAH1, may be related to vulnerability to vascular diseases such as hypertension and DM via the NO system.

Keywords: AGXT2, ADMA, Blood pressure, Casual blood sugar

\footnotetext{
* Correspondence: igajunichi@hotmail.com

${ }^{1}$ Department of Neuropsychiatry, Molecules and Function, Ehime University Graduate School of Medicine, Shitsukawa, Toon, Ehime 791-0295, Japan

Full list of author information is available at the end of the article
}

(c) The Author(s). 2021 Open Access This article is licensed under a Creative Commons Attribution 4.0 International License, which permits use, sharing, adaptation, distribution and reproduction in any medium or format, as long as you give appropriate credit to the original author(s) and the source, provide a link to the Creative Commons licence, and indicate if changes were made. The images or other third party material in this article are included in the article's Creative Commons licence, unless indicated otherwise in a credit line to the material. If material is not included in the article's Creative Commons licence and your intended use is not permitted by statutory regulation or exceeds the permitted use, you will need to obtain permission directly from the copyright holder. To view a copy of this licence, visit http://creativecommons.org/licenses/by/4.0/ The Creative Commons Public Domain Dedication waiver (http://creativecommons.org/publicdomain/zero/1.0/) applies to the data made available in this article, unless otherwise stated in a credit line to the data. 


\section{Background}

Cardiovascular disease (CVD) is one of the reasons for premature death, which is estimated from $4 \%$ in highincome countries to $42 \%$ in low-income countries [1]. The risk factor for developing CVD has been investigated but still unclear. Alanine:glyoxylate aminotransferase 2 (AGXT2; EC 2.6.1.44) is the only enzyme that has the ability to metabolize the R-form of 3-aminoisobutyrate (R-3AIB) with pyruvate to 2-methyl-3-oxopropanoate and Lalanine [2]. Interestingly, AGXT2 activity depends on the individual's genetic background; $30-40 \%$ of Japanese people show a lack of AGXT activity, compared with only $10 \%$ of individuals with European ancestry [2]. It has been shown that AGXT2 activity is decided by four functional single nucleotide polymorphisms (SNPs): rs37370 [3], rs37369 [4], rs180749 [5], and rs16899974 [6]. Furthermore, we previously found that the CAAA haplotype predicted by these four functional SNPs is strongly associated with loss of function in AGXT2 activity [7].

AGXT2 is also capable of metabolizing asymmetric dimethylarginine (ADMA), a unique methyl amino acid that competitively inhibits the nitric oxide synthase (NOS) family. ADMA is also metabolized by diaminoarginine dimethylaminohydrolase 1 (DDAH1; EC 3.5.3.18). Numerous studies have shown the association between serum/ plasma ADMA levels and several diseases, including hypertension [8], congestive heart failure [9], chronic kidney disease [10], atherosclerosis [11], type 2 diabetes mellitus (DM) [12], as well as the human metabolome [3]. In addition, the variants of two enzymes have been shown to be associated with several vascular conditions and diseases (AGXT2: carotid atherosclerosis [7], atrial fibrillation and ischemic stroke [13], and coronary heart disease [14]; $D D A H 1$ : hypertension [15] and T2DM [16]).

Considering that Agxt2 knockout (KO) mice show elevated levels of ADMA in circulation with reduced NO concentration and hypertension [17], it is possible that AGXT2 is a regulator of blood pressure (BP) even in humans. AGXT2 is known to be expressed abundantly in the liver and kidney (RNA-seq data in GeneCards), and four functional SNPs of AGXT2 may affect the liver and kidney functions of AGXT2 enzyme activity. To elucidate these points, we conducted the present study as follows. First, we determined the genotypes of four functional SNPs of $A G X T 2$, as well as one functional polymorphism with four SNPs of DDAH1, in 750 subjects (aged > 65 years) recruited using the complete enumeration survey (census) method in the Nakayama study. Second, we tested whether plasma ADMA concentrations were regulated by $A G X T 2$ and/or $D D A H 1$. Lastly, we examined whether the AGXT2 and DDAH1 genotypes were associated with clinical demographical and biological data such as BP and blood sugar using multiple regression analysis.

\section{Methods}

\section{Subjects recruited from the Nakayama study}

Nakayama is a rural community in Iyo city, Ehime Prefecture, Japan (2655 residents). This study was conducted with all older people aged > 65 years and living at home in Nakayama town. The subjects $(927 / 1512,61.3 \%)$ were recruited between January 2017 and April 2018. After excluding patients with dementia, 750 subjects were included in this study. Systolic blood pressure (SBP) and diastolic blood pressure (DBP) were measured three times, and the average values were used. All subjects were of unrelated Japanese origin and signed written informed consent forms approved by the institutional ethics committees of Ehime University Graduate School of Medicine (No. 1901009).

\section{Subjects in the urinary excretion of R-3-AIB and plasma ADMA concentration studies}

Eighty-five unrelated Japanese subjects (41 males, 44 females; mean age $=48.3 \pm 21.0$ years) were recruited from Ehime Prefecture, Japan, to analyze the association between urinary excretion of R-3-AIB and the CAAC $A G X T 2$ haplotype $[5,7]$. The cohort consisted of volunteer hospital workers, students, and citizens living in the same prefecture who did not have any urinary disorders as judged by blood examination. In addition to these 85 participants, 78 subjects were randomly selected from the Nakayama study to investigate whether ADMA concentrations were associated with four functional SNPs and the CAAA haplotype of $A G X T 2$.

\section{Blood sampling and DNA isolation}

Blood was collected into an EDTA tube. Subsequently, the plasma was collected as supernatant after centrifuging at $2000 \mathrm{rpm}$ for $10 \mathrm{~min}$ and submitted to LSI Medience Corporation (Tokyo, Japan) to examine the biochemical data. Casual blood sugar (CBS) was judged based on the interview of eating habits and measured by the hexokinase/G6PD method. In addition, a blood cell component (remaining precipitation) was used for DNA isolation from whole blood leukocytes. Briefly, DNA was isolated from frozen white blood cells using a blood mini-kit with QIAcube (Qiagen, Tokyo, Japan) and stored at $4{ }^{\circ} \mathrm{C}$.

\section{Genotyping and haplotype prediction}

Genotyping of $A G X T 2$ and DDAH1 SNPs was conducted using the TaqMan 5 '-exonuclease allelic discrimination assay (AGXT2: Assay IDs: rs37369; C__11162986_1, rs37370; C_1018750_1, rs180749; C_1018795_1, and rs16899974; C_25742181_10, respectively, and DDAH1: Assay IDs: rs307894; C__2518300_20, rs669173; C_ 658778_10, rs997251; C_2518388_10, and rs13373844; C_1406532_10, respectively)] using the StepOnePlus real-time PCR system (Applied Biosystems, Foster City, 
CA). In addition, 4-nucleotide (GCGT) insertion/deletion ( $4 \mathrm{~N}$ ins/del) on the promoter region of $D D A H 1$ was determined by a custom TaqMan probe assay, as previously described (20167924). The haplotype within rs37370, rs37369, rs180749, and rs16899974 was predicted by SNPAlyze (Dynacom, Tokyo, Japan), which could estimate the individual diplotype [18].

\section{ADMA concentrations}

An enzyme-linked immunoassay (ADMA Xpress ELIS; KR7860, Immundiagnostik, UK) was used to measure ADMA concentrations according to the manufacturer's protocol.

\section{Statistical analysis}

The Kolmogorov-Smirnov test of normality was conducted to determine whether the distribution was nonnormal. The average difference among the two groups was tested by Student's $t$-test or the Mann-Whitney $U$ test, and the average difference among the three groups was tested by one-way analysis of variance (ANOVA) or the Kruskal-Wallis test according to the normality. In addition to these analyses, multiple regression analysis and analysis of covariance (ANCOVA) were conducted using SPSS 22.0 (IBM Japan, Tokyo, Japan). Multicollinearity was determined according to the following criterion: a variance inflation factor $>10$. $\mathrm{D}^{\prime}$ for considering linkage disequilibrium (LD) was calculated by SNPAlyze (Dynacom), and the LD between two SNPs was set as more than $\mathrm{D}^{\prime}=0.9$. The estimations of the HardyWeinberg equilibrium (HWE) and minor allele frequency were conducted using Haploview software (version 4.2; Cambridge, MA, USA). Statistical significance was set at the $95 \%$ level $(p=0.05)$. The missing values for genotyping analysis were calculated by omission.

\section{Results}

\section{Subjects recruited from the Nakayama study}

The demographic data and biochemical data are shown in Tables 1 and 2, respectively. Because the participants were older, high rates of lifestyle diseases were found (e.g., hypertension: 560/749, 74.8\%; DM: 120/743, 16.2\%; kidney disease: 171/750, 22.8\%).

\section{Genotyping and haplotype analysis}

The genotyping rates were $>90.0 \%$ across $A G X T 2$ and $D D A H 1$ SNPs, and none of the $p$ values for the HWE reached statistical significance (Supplemental Table 1). In terms of LD, each pair of the four SNPs in AGXT2 was not in linkage equilibrium when considering $D^{\prime}$ values. However, the following five pairs in $D D A H 1$ had linkage equilibrium, as shown in Supplemental Table 2: rs3087894 and rs669173, $D^{\prime}=-1 ; \quad$ rs3087894 and rs997251, $D^{\prime}=0.9961 ;$ rs3087894 and rs13373844, $D^{\prime}=$
Table 1 Demographic and biochemical data from the Nakayama study

\begin{tabular}{lll}
\hline Parameters & Available number & Values \\
\hline Demographic data & & 750 \\
N & & $77.0 \pm 7.6$ \\
Age (years) & 750 & $309: 441$ \\
Sex (male:female) & 750 & $151.7 \pm 9.6$ \\
Height (cm) & 746 & $54.6 \pm 10.6$ \\
Weight (kg) & 747 & $23.6 \pm 3.3$ \\
BMl (kg/m²) & 746 & \\
Education (years) & 743 & $1: 33$ \\
1: $<6$ & & $2: 324$ \\
2: 7-9 & & $3: 323$ \\
3: 10-12 & & $4: 63$ \\
4: $\geq 13$ & & $137.8 \pm 15.6$ \\
Systolic blood pressure (mmHg) & 749 & $76.7 \pm 9.9$ \\
Diastolic blood pressure (mmHg) & 749 & $70.3 \pm 12.0$ \\
Heart rate (per min) & 748 & 560 \\
Hypertension & 749 & 120 \\
Diabetes mellitus & 743 & 44 \\
Liver disease & 748 & 171 \\
Kidney disease & 750 & 87 \\
Depression & 748 & 32 \\
Brain attack (past history of stroke) & 748 & \\
Head injury & 748 & \\
Current alcohol drinking & 724 & \\
Current smoking status & 723 & \\
\hline & & \\
\hline
\end{tabular}

- 1; rs669173 and rs997251, $\mathrm{D}^{\prime}=-0.9089$; and rs669173 and rs13373844, $\mathrm{D}^{\prime}=0.9171$. Therefore, only rs 997251 and rs13373844 within DDAH1 SNPs were used in the multiple regression analysis. As a result of the predicted haplotype of AGXT2 SNPs (rs37370, rs37369, rs180749, rs16899974), the highest rate was found in the CAAA haplotype (0.2429; Supplemental Table 3), which was the same finding as that in our previous study [7].

\section{Urinary excretion of D-AIB and plasma ADMA concentration}

None of the 85 subjects had two CAAC haplotypes. Therefore, the average difference was tested between two groups (number of CAAC haplotypes $=0$ or 1 ). Urinary R3 -AIB excretion was significantly higher in subjects with one CAAC haplotype than in those without a CAAC haplotype ( $p=0.004$; Supplemental Figure 1).

Plasma ADMA concentrations were significantly elevated in the AA genotype of rs187049 compared with the GG genotype $(p=0.001$; overall $p$ value $=0.001)$, and in subjects with two CAAA haplotypes compared with those without a CAAA haplotype $(p=0.033$; overall $p$ 
Table 2 Demographic and biochemical data from the Nakayama study

\begin{tabular}{lll}
\hline Parameters & Available number & Values \\
\hline Biochemical data & & \\
Albumin (g/dL) & 750 & $4.19 \pm 0.34$ \\
Total bilirubin (mg/dL) & 750 & $0.72 \pm 0.26$ \\
AST (IU/L) & 750 & $25.5 \pm 8.51$ \\
ALT (IU/L) & 750 & $18.9 \pm 11.7$ \\
LDH (IU/L) & 750 & $220.0 \pm 40.3$ \\
Y-GTP (IU/L) & 750 & $29.8 \pm 37.3$ \\
CPK & 750 & $121.1 \pm 70.5$ \\
Total cholesterol (mg/dL) & 750 & $196.7 \pm 31.8$ \\
LDL cholesterol (mg/dL) & 750 & $110.8 \pm 26.7$ \\
HDL cholesterol (mg/dL) & 750 & $59.0 \pm 26.7$ \\
Fasting blood glucose (mg/dL) & 24 & $102.1 \pm 42.0$ \\
Casual blood glucose (mg/dL) & 726 & $117.4 \pm 45.6$ \\
HbA1c (\%) & 750 & $5.85 \pm 0.82$ \\
BUN (mg/dL) & 750 & $17.2 \pm 4.93$ \\
Creatinine (mg/dL) & 750 & $0.76 \pm 0.26$ \\
eGFR (mL/min/1.73m²) & 750 & $66.7 \pm 12.0$ \\
Na (mEq/L) & 750 & $140.8 \pm 2.32$ \\
K (mEq/L) & 750 & $5.08 \pm 0.67$ \\
Cl (mEq/L) & 750 & $102.9 \pm 2.68$ \\
\hline ALT a & &
\end{tabular}

$A L T$ alanine aminotransferase, $A S T$ aspartate transaminase, $B M I$ body mass index, $B U N$ blood urea nitrogen, $C P K$ creatine phosphokinase, eGFR estimated glomerular filtration rate, $\gamma$-GTP $\gamma$-glutamyl transpeptidase, $H D L$ high density lipoprotein, $L D H$ lactate dehydrogenase, $L D L$ low-density lipoprotein

value $=0.083)$, as shown in Fig. 1 . No significant changes were observed in $\operatorname{rs} 37370(p=0.222), \operatorname{rs} 37369(p=$ $0.238)$, or rs16899974 $(p=0.986)$. Additionally, no significant changes were found in DDAH1 variants (rs997251, $p=0.559 ;$ rs13373844, $p=0.395 ; 4 \mathrm{~N}$ ins/del, $p=0.503)$, as shown in Supplemental Figure 2 .

\section{Multiple regression analysis and ANCOVA}

The details of each parameter used in the multiple regression analysis are explained in Supplemental Table 4.

\section{Systolic blood pressure (SBP) and diastolic blood pressure (DBP)}

The number of CAAA haplotypes in AGXT2 was significantly associated with both SBP $(p=0.031)$ and DBP $(p=0.028)$, as shown in Tables 3 and 4 . In addition, rs16899974 was significantly associated with DBP ( $p=$ 0.040). Other than the AGXT2 and DDAH1 genotypes, the following parameters were significantly associated with SBP (sex $[p<0.001]$, body mass index [BMI; $p=$ $0.020]$, depression $[p=0.026]$, head injury $[p=0.022]$, and total cholesterol $[p=0.003]$ ) and with DBP (age $[p=0.03]$, sex $[p=0.002]$, BMI $[p=0.002]$, kidney disease $[p=0.004]$, depression $[p=0.042]$, and total cholesterol $[p=0.026])$. The individual SNPs of DDAH1 was not significantly associated with SBP and DBP (Supplemental Table $5 \mathrm{a}$ and $5 \mathrm{~b}$ ). The average differences in SBP and DBP among the number of CAAA haplotypes $(0,1$, or 2$)$ were tested by setting all values except the number of CAAA haplotypes as covariates. Both SBP $(p=0.546)$ and DBP $(p=0.767)$ showed a trend, but did not reach the level of statistical significance. The average DBP value was not significantly changed among the rs16899974 genotype $(p=0.431)$. Subsequently, we conducted liner regression analysis in non-HT and HT subjects separately with including antihypertensive drugs to investigate how AGXT2 SNPs and haplotype affect SBP and DBP when considering disease state and drugs affect. For SBP, the number of CAAA haplotypes showed a trend, but did not reach the level of statistical significance $(p=0.059)$ in HT subjects. In term of DBP, there were significant associations in $\operatorname{rs} 16899974(p=$ $0.009)$ and the number of CAAA haplotypes $(p=0.008)$ in HT subjects (Supplemental Table 6a and 6b).

\section{Aspartate aminotransferase (AST) and alanine aminotransferase (ALT)}

The rs16899974 genotype was significantly associated with $\operatorname{AST}(p=0.026)$ and $\operatorname{ALT}(p=0.039)$, as shown in Tables 5 and 6 . Furthermore, the number of CAAA haplotypes was significantly associated with AST $(p=0.011)$, but not with ALT $(p=0.083)$. Other than the AGXT2 and $D D A H 1$ genotypes, the following parameters were significantly associated with AST (hypertension $[p=$ $0.029]$ ) and ALT (age $[p<0.001]$, BMI $[p=0.004]$, DM $[p=0.005]$, and depression $[p<0.001])$. The individual SNPs of DDAH1 was not significantly associated with AST and ALT (Supplemental Table 7a and 7b). The average differences in AST and ALT among the number of CAAA haplotypes and the rs16899974 genotype were tested by setting all values as covariates except for the number of CAAA haplotypes and rs16899974, respectively. No significant changes were seen for AST (rs1689974, $p=0.847$; CAAA haplotype $p=0.300$ ) or ALT (rs16899974, $p=0.915)$.

\section{Blood urea nitrogen (BUN) and creatinine}

The rs37370 genotype was significantly associated with BUN $(p=0.020)$ and creatinine $(p=0.023)$, as shown in Tables 7 and 8 . The rs180749 genotype also reached a significant level in BUN $(p=0.032)$. Other than the AGXT2 and $D D A H 1$ genotypes, the following parameters were significantly associated with BUN (age $[p<0.001]$ ) and creatinine (age $[p<0.001]$, sex $[p<0.001]$, and BMI $[p=$ $0.014])$. The individual SNPs of $D D A H 1$ was not significantly associated with BUN and creatinine (Supplemental Table $8 \mathrm{a}$ and $8 \mathrm{~b}$ ). The average differences in BUN among 


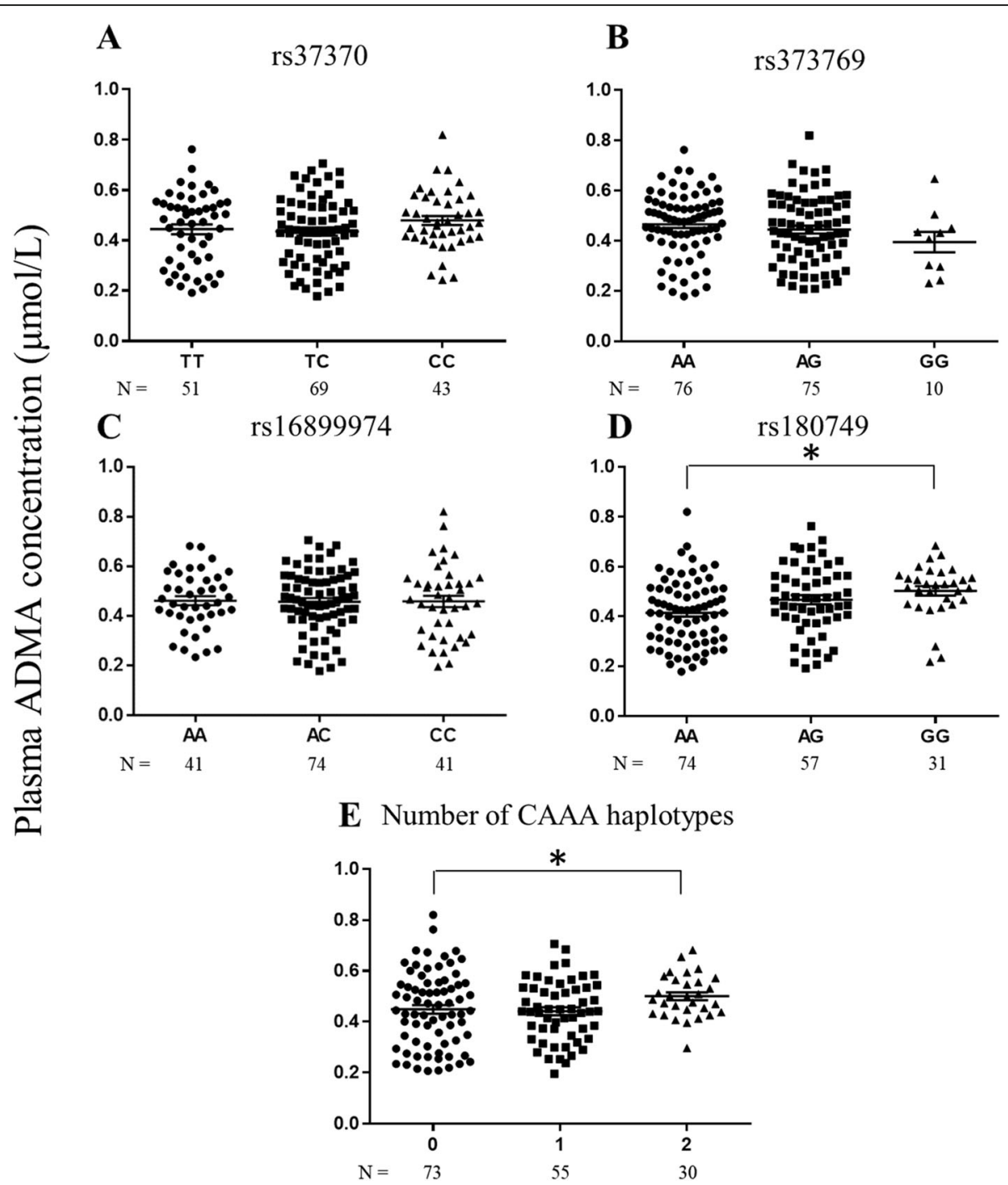

Fig. 1 Effects of functional SNPs and CAAA haplotype in AGXT2 on ADMA concentrations. Average ADMA concentrations were tested by oneway ANOVA or the Kruskal-Wallis test among the a rs37370 ( $p=0.222)$, b rs37369 ( $p=0.238)$, c rs16899974 ( $p=0.986)$, d rs 180749 ( $p=0.001)$, and e CAAA haplotypes $(p=0.083)$. The horizontal bar represents the mean \pm standard error. Statistical significance based on a post hoc test among the two groups is indicated by an asterisk $\left(^{*}\right)$. CAAA was predicted by each allele of the four SNPs as follows: rs37370 (c), rs37369 (a), rs180749 (a), and rs16899974 (a). SNP, single nucleotide polymorphism

the rs37370 and rs180749 genotypes were tested by setting all values as covariates except the rs37370 and rs180749 genotypes, respectively. The average BUN value was not significantly changed in the rs37370 genotype $(p=0.675)$ and the rs180749 genotype $(p=0.158)$.

\section{Casual blood sugar (CBS)}

The rs16899974 genotype was significantly associated with CBS $(p=0.028)$, as shown in Table 9. The number of CAAA haplotypes showed a trend, but did not reach the level of statistical significance $(p=0.086)$. Other than the $A G X T 2$ and DDAH1 genotypes, BMI was significantly associated with CBS $(p=0.013)$. The individual SNPs of DDAH1 was not significantly associated with CBS (Supplemental Table 9). The average difference in CBS for the rs16899974 genotype was tested by setting all values as covariates except the rs1689974 genotype. The average CBS value showed a trend for the rs16899974 genotype, but did not reach the level of statistical significance $(p=0.310)$. Subsequently, we conducted liner regression analysis in non-DM and DM subjects separately with including antidiabetic drugs to investigate how AGXT2 SNPs and haplotype affect CBS when considering disease state and drugs affect. There were significant associations in $\operatorname{rs} 16899974(p=0.026)$ and the number of CAAA haplotypes $(p=0.007)$ in nonDM subjects (Supplemental Table 10). 
Table 3 Multiple regression analysis for systolic blood pressure within AGXT2 SNPs and haplotype

\begin{tabular}{lll}
\hline Parameters & $\boldsymbol{\beta}$ & $\boldsymbol{P}$ value \\
\hline rs37370 & -0.081 & 0.17 \\
rs37369 & 0.036 & 0.45 \\
rs180749 & 0.041 & 0.36 \\
rs16899974 & 0.047 & 0.38 \\
CAAA haplotype & 0.154 & 0.031 \\
Age & 0.082 & 0.075 \\
Sex & -0.174 & $<0.001$ \\
BMl & 0.088 & 0.020 \\
Education & 0.017 & 0.69 \\
Diabetes mellitus & 0.069 & 0.069 \\
Kidney disease & -0.019 & 0.64 \\
Depression & -0.083 & 0.026 \\
Brain attack & -0.023 & 0.54 \\
Head injury & -0.086 & 0.022 \\
Current alcohol drinking & 0.042 & 0.31 \\
Current smoking status & -0.055 & 0.17 \\
Total cholesterol & 0.119 & 0.003 \\
\hline BMI body mass index brain attack (defined & as &
\end{tabular}

$B M I$ body mass index, brain attack (defined as past history of stroke)
Table 5 Multiple regression analysis for AST within AGXT2 SNPs and haplotype

\begin{tabular}{lll}
\hline Parameters & $\boldsymbol{\beta}$ & $\boldsymbol{P}$ value \\
\hline rs37370 & 0.044 & 0.46 \\
rs37369 & -0.032 & 0.50 \\
rs180749 & -0.18 & 0.69 \\
rs16899974 & -0.122 & 0.026 \\
CAAA haplotype & -0.185 & 0.011 \\
Age & -0.066 & 0.16 \\
Sex & -0.038 & 0.36 \\
BMl & -0.027 & 0.49 \\
Education & -0.007 & 0.88 \\
Hypertension & 0.084 & 0.029 \\
Diabetes mellitus & 0.036 & 0.35 \\
Kidney disease & -0.018 & 0.66 \\
Depression & 0.071 & 0.062 \\
Current alcohol drinking & 0.025 & 0.55 \\
Current smoking status & 0.030 & 0.47 \\
Total cholesterol & -0.054 & 0.19 \\
\hline AST aspata amingtrat &
\end{tabular}

AST aspartate aminotransferase, $B M I$ body mass index
Table 4 Multiple regression analysis for diastolic blood pressure within AGXT2 SNPs and haplotype

\begin{tabular}{lll}
\hline Parameters & $\boldsymbol{\beta}$ & $\boldsymbol{P}$ value \\
\hline rs37370 & -0.065 & 0.26 \\
rs37369 & 0.060 & 0.20 \\
rs180749 & -0.034 & 0.43 \\
rs16899974 & 0.109 & 0.040 \\
CAAA haplotype & 0.155 & 0.028 \\
Age & -0.096 & 0.034 \\
Sex & -0.127 & 0.002 \\
BMl & 0.114 & 0.002 \\
Education & 0.031 & 0.45 \\
Diabetes mellitus & -0.072 & 0.054 \\
Kidney disease & -0.115 & 0.004 \\
Depression & -0.075 & 0.042 \\
Brain attack & 0.014 & 0.71 \\
Head injury & -0.061 & 0.098 \\
Current alcohol drinking & 0.039 & 0.33 \\
Current smoking status & -0.022 & 0.58 \\
Total cholesterol & 0.087 & 0.026 \\
\hline BMI body mass & &
\end{tabular}

$B M I$ body mass index, brain attack (defined as past history of stroke)
Table 6 Multiple regression analysis for ALT within AGXT2 SNPS and haplotype

\begin{tabular}{lll}
\hline Parameters & $\boldsymbol{\beta}$ & $\boldsymbol{P}$ value \\
\hline rs37370 & 0.023 & 0.68 \\
rs37369 & 0.050 & 0.27 \\
rs180749 & 0.007 & 0.87 \\
rs16899974 & -0.108 & 0.039 \\
CAAA haplotype & -0.119 & 0.083 \\
Age & -0.161 & $<0.001$ \\
Sex & -0.063 & 0.11 \\
BMl & 0.107 & 0.004 \\
Education & 0.041 & 0.32 \\
Hypertension & 0.071 & 0.052 \\
Diabetes mellitus & 0.103 & 0.005 \\
Kidney disease & -0.048 & 0.22 \\
Depression & 0.147 & $<0.001$ \\
Current alcohol drinking & -0.010 & 0.81 \\
Current smoking status & 0.069 & 0.079 \\
Total cholesterol & -0.032 & 0.41 \\
\hline
\end{tabular}


Table 7 Multiple regression analysis for BUN within AGXT2 SNPS and haplotype

\begin{tabular}{lll}
\hline Parameters & $\boldsymbol{\beta}$ & $\boldsymbol{P}$ value \\
\hline rs37370 & 0.134 & 0.020 \\
rs37369 & 0.025 & 0.59 \\
rs180749 & 0.094 & 0.032 \\
rs16899974 & 0.039 & 0.46 \\
CAAA haplotype & -0.063 & 0.37 \\
Age & 0.294 & $<0.001$ \\
Sex & -0.060 & 0.14 \\
BMl & -0.008 & 0.82 \\
Education & 0.069 & 0.097 \\
Hypertension & -0.012 & 0.75 \\
Diabetes mellitus & 0.008 & 0.82 \\
Liver disease & 0.009 & 0.81 \\
Depression & -0.046 & 0.21 \\
Current alcohol drinking & 0.011 & 0.78 \\
Current smoking status & -0.020 & 0.62 \\
Total cholesterol & 0.003 & 0.93 \\
\hline BMI body mass index, BuN blood
\end{tabular}

$B M I$ body mass index, BUN blood urea nitrogen

\section{Discussion}

To our knowledge, this is the first study to report an association between the AGXT2 genotype and both BP and biochemical data. The recruitment method used in this study was the census method (within those aged > 65 years), which aimed to measure all members of the

Table 8 Multiple regression analysis for creatinine within AGXT2 SNPs and haplotype

\begin{tabular}{lll}
\hline Parameters & $\boldsymbol{\beta}$ & $\boldsymbol{P}$ value \\
\hline rs37370 & 0.119 & 0.023 \\
rs37369 & -0.002 & 0.97 \\
rs180749 & 0.011 & 0.79 \\
rs16899974 & 0.028 & 0.56 \\
CAAA haplotype & -0.070 & 0.27 \\
Age & 0.234 & $<0.001$ \\
Sex & -0.424 & $<0.001$ \\
BMl & 0.084 & 0.014 \\
Education & 0.005 & 0.89 \\
Hypertension & 0.043 & 0.20 \\
Diabetes mellitus & -0.031 & 0.35 \\
Liver disease & 0.037 & 0.28 \\
Depression & -0.035 & 0.30 \\
Current alcohol drinking & 0.062 & 0.091 \\
Current smoking status & -0.051 & 0.16 \\
Total cholesterol & -0.018 & 0.61 \\
\hline BM body mass index & &
\end{tabular}

BMI body mass index
Table 9 Multiple regression analysis for casual blood sugar within AGXT2 SNPs and haplotype

\begin{tabular}{lll}
\hline Parameters & $\boldsymbol{\beta}$ & $\boldsymbol{P}$ value \\
\hline rs37370 & 0.007 & 0.91 \\
rs37369 & -0.035 & 0.47 \\
rs180749 & 0.007 & 0.88 \\
rs16899974 & -0.122 & 0.028 \\
CAAA haplotype & -0.126 & 0.086 \\
Age & -0.033 & 0.46 \\
Sex & -0.026 & 0.53 \\
BMI & 0.097 & 0.013 \\
Education & -0.009 & 0.84 \\
Hypertension & 0.033 & 0.39 \\
Liver disease & $<0.001$ & 0.99 \\
Depression & 0.006 & 0.87 \\
Current alcohol drinking & 0.071 & 0.088 \\
Current smoking status & 0.073 & 0.078 \\
Total cholesterol & -0.055 & 0.18 \\
\hline BMI body mass index & &
\end{tabular}

$B M I$ body mass index

whole target population in Nakayama town. The advantage of the census method is its accuracy in terms of recruiting a pure population as compared with a sampling method using each unit of the population because the effect of community background is theoretically excluded. We recruited a total of 927 subjects $(61.3 \%$ of the whole population aged $>65$ years). Among the subjects' DNA samples, the genotypes of four functional SNPs in AGXT2 and associated haplotypes were successfully measured, and the results, such as allele frequencies, were almost the same as those in our previous studies [5, 7]. In terms of DDAH1 SNPs and $4 \mathrm{~N}$ ins/del, the allele frequencies of these genotypes were also similar to those in the dbSNP database (https://www.ncbi. nlm.nih.gov/snp/) and a previous report [16]. In addition, subjects with two CAAA haplotypes had higher concentrations of ADMA than those without the CAAA haplotype. This result strongly matched that each allele $(\mathrm{rs} 37370=\mathrm{C}, \quad \mathrm{rs} 37369=\mathrm{A}, \quad \mathrm{rs} 16899974=\mathrm{A}, \quad$ and rs180749= A) of the CAAA haplotype is relevant to the loss of function $[5,7]$, and subjects who have the CAAA haplotype showed high R-3-AIB excretion [7]. However, we have to consider that ADMA concentrations were significantly higher in the GG genotype (gain of function) than in the AA genotype (loss of function) of rs180749. Because rs180749 is one of the functional SNPs that regulates AGXT2 activity, it is hard to reach a definite conclusion without considering other functional SNPs. Therefore, we think that the CAAA haplotype is a stronger predictor of AGXT2 activity than are functional SNPs. Regarding $D D A H 1$, a previous study reported 
elevated plasma ADMA concentrations in subjects with an insertion allele in $4 \mathrm{~N}$ ins/del [19]; however, no significant results were found in this study. As only three subjects in the present study had the ins/ins genotype, more subjects are needed to clarify this point. Based on these results and insights, four functional SNPs in AGXT2 (rs37370, rs37369, rs180749, and rs16899974), the CAAA haplotype predicted by those four SNPs, two SNPs (rs997251 and rs13373844), and $4 \mathrm{~N}$ ins/del in $D D A H 1$, were considered to affect $\mathrm{BP}$ and several biochemical tests after excluding DDAH1 SNPs with LD.

The more subjects who had a CAAA haplotype, the higher SBP and DBP values were confirmed. Our previous study showed that the CAAA haplotype is associated with a loss of function, which was revealed by measuring urine R-3-AIB [7]. Contrary to the result regarding the CAAA haplotype when considering AGXT2 activity, the CC genotype of rs16899974 (gain of function) was associated with elevated DBP. Because the CAAC haplotype changed from the A allele to the C allele on rs16899974, it still plays a role in the loss of function, and the effect of the CAAA haplotype on AGXT2 activity is more effective than that on rs16899974. Considering that AGXT2 plays a role in metabolizing ADMA, a decrease in NO production is induced among people who have the CAAA haplotype. Indeed, decreased plasma NO was found in Agxt2 KO mice, which also showed elevated plasma ADMA concentrations and mean arterial pressure as measured in the carotid artery [17]. Doğan et al. (2018) reported a correlation between serum ADMA levels and SBP [20]. In addition, it has been shown that high ADMA levels in plasma/serum are relevant to the exaggerated $\mathrm{BP}$ response to exercise [8], congestive heart failure [9], and coronary atherosclerosis [11], and the AGXT2 genotypes are associated with atrial fibrillation and ischemic stroke [13] and coronary heart disease [14]. Collectively, we hypothesize that the loss of AGXT2 function induces elevated SBP and DBP through the dysregulation of the NOS/ADMA pathway, which means that we can estimate the risk of cardiovascular diseases by detecting AGXT2 SNPs.

The loss of AGXT2 function predicted by the CAAA haplotype contributes to a decrease in AST. Conversely, the loss of AGXT2 function predicted by rs16899974 contributes to elevated AST and ALT. AGXT2 is mainly present in the mitochondria of hepatocytes [21]. A previous isotope study showed the existence of $\alpha-k e t o-\delta-\left(N^{G}\right.$, $\mathrm{N}^{\mathrm{G}}$-dimethylguanidino) valeric acid (DMGV) in the liver, which is a metabolite of ADMA [22]. These lines of evidence suggest that metabolism from ADMA to DMVG occurs even in the liver. Growing evidence has shown that elevated plasma ADMA levels are found in patients with severe acute alcoholic hepatitis [23] and acute liver failure [24]. However, the functions of AGXT2 and
ADMA in the liver are still unknown under both the normal condition and disease state.

Higher BUN values were found in the CC genotype of rs37370 (loss of function) and GG genotype of rs180749 (gain of function), which are discrepant results in terms of the predicted AGXT2 function. Within kidney tissues, AGXT2 is strongly expressed in the renal proximal tubular epithelium [21]. It has been shown that $H N F 4 \alpha$, a nuclear transcription factor that can work by binding on promoter regions of $A G X T 2$, is co-localized in the renal proximal tubular epithelium [21]. However, AGXT2 function itself is still unknown, even though AGXT2 expression is driven in kidney tissue.

Surprisingly, the loss of function of AGXT2 activity predicted by rs16899974 (AA genotype) was associated with elevated CBS. Several reports have shown that elevated ADMA concentrations are found in subjects with type $1 \mathrm{DM}[25,26]$, type $2 \mathrm{DM}[12]$ and poor glycemic control [27]. Furthermore, the circulating level of ADMA is relevant to several DM-related complications, such as microvascular complications [28, 29], cardiovascular complications [30], and diabetic nephropathy [31]. Given that subjects who have the AA genotype show higher ADMA concentrations [32], the elevated CBS in the AA genotype of $A G X T 2$ is caused by changes in ADMA levels. It may be therefore possible to predict type 2 DM complications and whether CBS tends to rise easily in patients based on rs16899974.

This study had several limitations. First, the number of samples was relatively small as a population-based study, and there are some missing values. In this sense, type I error is not completely excluded. However, we used the census method to determine the clinical test items in a unit (Nakayama town) accurately, which is a strong advantage compared with the sampling method. Second, several discrepant results were found in the results of the biochemical tests on liver and kidney functions. A possible cause for this was that we could not determine AGXT2 activity by one SNP because four functional SNPs are assumed to regulate AGXT2 activity. In addition, AGXT2 functions in the liver and kidney are still unknown, even though these expressions were confirmed [21]. Lastly, we did not include information on what and how much the recruited subjects had eaten before blood sampling for CBS. Taken together, further studies with large samples and more detailed information on the subjects' backgrounds are needed to clarify these points and reproducibility.

\section{Conclusions}

In conclusion, we revealed that the loss of AGXT2 function predicted by the CAAA haplotype induces elevated ADMA concentrations. Elevated ADMA concentrations through the CAAA haplotype may increase SBP/DBP 
and CBS, respectively. Despite the fact that additional research is needed in terms of AGXT2 function in the kidney and liver, the four functional SNPs of AGXT2 and their haplotypes are useful tools for predicting hypertension, DM, and their related complications. When considering the loss of AGXT2 function depends on races, clinicians need to think through the genetic background in daily clinical practice.

\section{Abbreviations \\ $4 \mathrm{~N}$ ins/del: 4-nucleotide (GCGT) insertion/deletion; ADMA: Asymmetric dimethylarginine; ALT: Alanine aminotransferase; ANOVA: One-way analysis of variance; AGXT2: Alanine:glyoxylate aminotransferase 2; AST: Aspartate aminotransferase; BMI: Body mass index; BP: Blood pressure; BUN: Blood urea nitrogen; CBS: Casual blood sugar; DBP: Diastolic blood pressure; \\ DDAH1: Diaminoarginine dimethylaminohydrolase 1; DM: Diabetes mellitus;

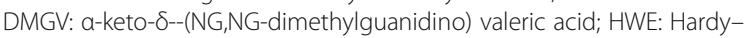 Weinberg equilibrium; KO: Knockout; LD: Linkage disequilibrium; NO: Nitric oxide; NOS: Nitric oxide synthase; R-3-AIB: R-form of 3-aminoisobutyrate; SBP: Systolic blood pressure; SNP: Single nucleotide polymorphisms}

\section{Supplementary Information}

The online version contains supplementary material available at https://doi. org/10.1186/s12864-021-07612-3.

Additional file 1: Figure S1. Average change in R-3-AIB based on CAAC haplotype. Average urinary R-3-AIB excretion values between subjects with one and no CAAC haplotypes were tested using the MannWhitney $U$ test $(p=0.004)$. The horizontal bar represents the mean \pm standard error. Statistical significance is indicated by an asterisk $\left(^{*}\right)$. R-3AIB, the R-form of 3-aminoisobutyrate; CAAC, was predicted by each allele of the four SNPs as follows: rs37370 (C), rs37369 (A), rs180749 (A), rs16899974 (C).

Additional file 2: Figure S2. Effects of DDAH1 variants in AGXT2 on ADMA concentrations. Average ADMA concentrations were tested by one-way ANOVA or the Kruskal-Wallis test among A) rs997251 ( $p=0.559)$, B) rs13373844 ( $p=0.395)$, and C) $-3964 \mathrm{~N}$ ins/del $(p=0.503)$. The horizontal bar represents mean \pm standard error. Ins, insertion; del, deletion.

Additional file 3: Table S1. Main characteristics of the select tagging SNPs in AGXT2 and DDAH1. Table S2. Linkage disequilibrium on the SNP results for AGXT2 and DDAH1. Table S3. Haplotype analysis for AGXT2 SNPS. Table S4. Explanation of each parameters used in the demographic data and multiple regression analysis. Table S5a. Association between Systolic Blood Pressure and SNPs in DDAH1. Table S5b. Association between Diastolic Blood Pressure and SNPs in DDAH1. Table S6a. Multiple regression analysis for systolic blood pressure in non-HT subjects within AGXT2 SNPs and haplotype. Table S6b. Multiple regression analysis for diastolic blood pressure in non-HT subjects within AGXT2 SNPs and haplotype. Table S7a. Association between AST and SNPs in DDAH1. Table S7b. Association between ALT and SNPs in DDAH1. Table S8a. Association between BUN and SNPS in DDAH1. Table S8b. Association between creatinine and SNPS in DDAH1. Table S9. Association between CBS and SNPS in DDAH1. Table S10. Multiple regression analysis for casual blood sugar within AGXT2 SNPs and haplotype.

\section{Acknowledgments}

We would like to thank Ms. Chiemi Onishi for her technical assistance. We also wish to thank Shinichiro Ochi, Yu Funahashi, and Hiroaki Mori for their technical advice.

\section{Declaration of interests}

No conflicts of interest.

\section{Authors' contributions}

YY conceptualized, generated, analyzed the data, and wrote manuscript. HK analyzed data. YY, TM, TY, AT, and HS collected data. JI interpreted the data and reviewed manuscript. SU edited the manuscript. All authors read and approved the final manuscript.

\section{Funding}

This study was supported by the Japan Agency for Medical Research and Development (dk0207025). This work was partially supported by a Health and Labour Science Research Grant from the Japanese Ministry of Health, Labour and Welfare and a Grant-in-Aid for Scientific Research from the Japanese Ministry of Education, Culture, Sports, Science and Technology (JSPS KAKENHI Grant No. 18H02752). All authors read and approved the final manuscript.

\section{Availability of data and materials}

The datasets used and/or analyzed during the current study are available from the corresponding author on reasonable request.

\section{Declarations}

\section{Ethics approval and consent to participate}

This study was conducted in accordance with the Declaration of Helsinki. All experimental protocols were approved by the institutional ethics committees of Ehime University Graduate School of Medicine (No. 1901009). Informed consent was obtained from all subjects.

\section{Consent for publication}

Not applicable.

\section{Competing interests}

All of authors have no competing interests.

\section{Author details}

${ }^{1}$ Department of Neuropsychiatry, Molecules and Function, Ehime University Graduate School of Medicine, Shitsukawa, Toon, Ehime 791-0295, Japan.

2Department of Neuropsychiatry, Zaidan Niihama Hospital, 13-47 Matsubara, Niihama, Ehime 792-0828, Japan.

Received: 30 December 2020 Accepted: 13 April 2021

Published online: 20 April 2021

\section{References}

1. Chittakath Shaima PVM, Shaheen NK. Cardiovascular diseases: traditional and non-traditional risk factors. J Med Allied Sci. 2016;6(2):46-51.

2. Kakimoto Y, Taniguchi K, Sano I. D-beta-aminoisobutyrate:pyruvate aminotransferase in mammalian liver and excretion of betaaminoisobutyrate by man. J Biol Chem. 1969;244(2):335-40.

3. Rhee EP, Ho JE, Chen MH, Shen D, Cheng S, Larson MG, et al. A genomewide association study of the human metabolome in a community-based cohort. Cell Metab. 2013;18(1):130-43.

4. Suhre K, Wallaschofski H, Raffler J, Friedrich N, Haring R, Michael K, et al. A genome-wide association study of metabolic traits in human urine. Nat Genet. 2011;43(6):565-9.

5. Yoshino $Y$, Abe $M$, Numata $S$, Ochi $S$, Mori $Y$, Ishimaru T, et al. Missense variants of the alanine:glyoxylate aminotransferase 2 gene are not associated with Japanese schizophrenia patients. Prog NeuroPsychopharmacol Biol Psychiatry. 2014;53:137-41.

6. Kittel A, Muller F, Konig J, Mieth M, Sticht H, Zolk O, et al. Alanine-glyoxylate aminotransferase 2 (AGXT2) polymorphisms have considerable impact on methylarginine and beta-aminoisobutyrate metabolism in healthy volunteers. PLoS One. 2014;9(2):e88544.

7. Yoshino Y, Kohara K, Abe M, Ochi S, Mori Y, Yamashita K, et al. Missense variants of the alanine: glyoxylate aminotransferase 2 gene correlated with carotid atherosclerosis in the Japanese population. J Biol Regul Homeost Agents. 2014;28(4):605-14.

8. Kayrak M, Bacaksiz A, Vatankulu MA, Ayhan SS, Taner A, Unlu A, et al. Association between exaggerated blood pressure response to exercise and serum asymmetric dimethylarginine levels. Circ J. 2010;74(6):1135-41.

9. Tang WH, Tong W, Shrestha K, Wang Z, Levison BS, Delfraino B, et al. Differential effects of arginine methylation on diastolic dysfunction and 
disease progression in patients with chronic systolic heart failure. Eur Heart J. 2008:29(20):2506-13.

10. Vallance $\mathrm{P}$, Leone A, Calver A, Collier J, Moncada S. Accumulation of an endogenous inhibitor of nitric oxide synthesis in chronic renal failure. Lancet. 1992;339(8793):572-5.

11. Mangiacapra F, Conte M, Demartini C, Muller O, Delrue L, Dierickx K, et al. Relationship of asymmetric dimethylarginine (ADMA) with extent and functional severity of coronary atherosclerosis. Int J Cardiol. 2016;220:629-33.

12. Abbasi F, Asagmi T, Cooke JP, Lamendola C, McLaughlin T, Reaven GM, et al. Plasma concentrations of asymmetric dimethylarginine are increased in patients with type 2 diabetes mellitus. Am J Cardiol. 2001;88(10):1201-3.

13. Seppala I, Kleber ME, Bevan S, Lyytikainen LP, Oksala N, Hernesniemi JA, et al. Associations of functional alanine-glyoxylate aminotransferase 2 gene variants with atrial fibrillation and ischemic stroke. Sci Rep. 2016;6:23207.

14. Zhou JP, Bai YP, Hu XL, Kuang DB, Shi RZ, Xiong Y, et al. Association of the AGXT2 V140l polymorphism with risk for coronary heart disease in a Chinese population. J Atheroscler Thromb. 2014;21(10):1022-30

15. Wang Z, Chen S, Zhang L, Lu G, Zhou C, Wang DW, et al. Association between variation in the genes DDAH1 and DDAH2 and hypertension among Uygur, Kazakh and Han ethnic groups in China. Sao Paulo Med J. 2016;134(3):205-10.

16. Zhu F, Zhou C, Wen Z, Wang DW. DDAH1 promoter $-3964 \mathrm{~N}$ insertion variant is associated with increased risk of type 2 diabetes in a genderdependent manner. Mol Genet Genomic Med. 2020;8(1):e1011.

17. Caplin B, Wang Z, Slaviero A, Tomlinson J, Dowsett L, Delahaye M, et al. Alanine-glyoxylate aminotransferase-2 metabolizes endogenous methylarginines, regulates $\mathrm{NO}$, and controls blood pressure. Arterioscler Thromb Vasc Biol. 2012;32(12):2892-900.

18. Awata T, Neda T, lizuka H, Kurihara S, Ohkubo T, Takata N, et al. Endothelial nitric oxide synthase gene is associated with diabetic macular edema in type 2 diabetes. Diabetes Care. 2004;27(9):2184-90.

19. Ding $H$, Wu B, Wang $H$, Lu Z, Yan J, Wang X, et al. A novel loss-of-function DDAH1 promoter polymorphism is associated with increased susceptibility to thrombosis stroke and coronary heart disease. Circ Res. 2010;106(6):1145-52.

20. Dogan I, Eser B, Ozkurt S, Yayar O, Ozgur B, Kayadibi H, et al. Serum ADMA, endothelial dysfunction, and atherosclerosis in hypervolemic hemodialysis patients. Turk J Med Sci. 2018;48(5):1041-7.

21. Jarzebska N, Georgi S, Jabs N, Brilloff S, Maas R, Rodionov RN, et al. Kidney and liver are the main organs of expression of a key metabolic enzyme alanine:glyoxylate aminotransferase 2 in humans. Atheroscler Suppl. 2019;40: 106-12.

22. Martens-Lobenhoffer J, Rodionov RN, Bode-Boger SM. Probing AGXT2 enzyme activity in mouse tissue by applying stable isotope-labeled asymmetric dimethyl arginine as substrate. J Mass Spectrom. 2012;47(12): 1594-600.

23. Mookerjee RP, Malaki M, Davies NA, Hodges SJ, Dalton RN, Turner C, et al. Increasing dimethylarginine levels are associated with adverse clinical outcome in severe alcoholic hepatitis. Hepatology. 2007;45(1):62-71.

24. Mookerjee RP, Dalton RN, Davies NA, Hodges SJ, Turner C, Williams R, et al. Inflammation is an important determinant of levels of the endogenous nitric oxide synthase inhibitor asymmetric dimethylarginine (ADMA) in acute liver failure. Liver Transpl. 2007;13(3):400-5.

25. Altinova AE, Arslan M, Sepici-Dincel A, Akturk M, Altan N, Toruner FB, Uncomplicated type 1 diabetes is associated with increased asymmetric dimethylarginine concentrations. J Clin Endocrinol Metab. 2007:92(5):1881-5.

26. Eid HM, Eritsland J, Larsen J, Arnesen H, Seljeflot I. Increased levels of asymmetric dimethylarginine in populations at risk for atherosclerotic disease. Effects of pravastatin. Atherosclerosis. 2003;166(2):279-84.

27. Can A, Bekpinar S, Gurdol F, Tutuncu Y, Unlucerci Y, Dinccag N. Dimethylarginines in patients with type 2 diabetes mellitus: relation with the glycaemic control. Diabetes Res Clin Pract. 2011;94(3):e61-4.

28. Ganz T, Wainstein J, Gilad S, Limor R, Boaz M, Stern N. Serum asymmetric dimethylarginine and arginine levels predict microvascular and macrovascular complications in type 2 diabetes mellitus. Diabetes Metab Res Rev. 2017;33(2):e2836.

29. Liu J, Li C, Chen W, He K, Ma H, Ma B, et al. Relationship between serum asymmetric dimethylarginine level and microvascular complications in diabetes mellitus: a meta-analysis. Biomed Res Int. 2019;2019:2941861.

30. Konya H, Miuchi M, Satani K, Matsutani S, Yano Y, Tsunoda T, et al. Asymmetric dimethylarginine, a biomarker of cardiovascular complications in diabetes mellitus. World J Exp Med. 2015;5(2):110-9.
31. Jayachandran I, Sundararajan S, Paramasivam P, Venkatesan B, Subramanian SC, Balasubramanyam M, et al. Association of circulatory asymmetric dimethylarginine (ADMA) with diabetic nephropathy in Asian Indians and its causative role in renal cell injury. Clin Biochem. 2017;50(15):835-42.

32. Amir M, Hassanein Sl, Abdel Rahman MF, Gad MZ. AGXT2 and DDAH-1 genetic variants are highly correlated with serum ADMA and SDMA levels and with incidence of coronary artery disease in Egyptians. Mol Biol Rep. 2018:45(6):2411-9.

\section{Publisher's Note}

Springer Nature remains neutral with regard to jurisdictional claims in published maps and institutional affiliations.

\section{Ready to submit your research? Choose BMC and benefit from:}

- fast, convenient online submission

- thorough peer review by experienced researchers in your field

- rapid publication on acceptance

- support for research data, including large and complex data types

- gold Open Access which fosters wider collaboration and increased citations

- maximum visibility for your research: over $100 \mathrm{M}$ website views per year

At BMC, research is always in progress.

Learn more biomedcentral.com/submissions 